\title{
Searching for evolutions from pure states into mixed states with entangled neutral kaons
}

\section{Journal Article}

\section{Author(s):}

Gerber, Hans-Jürg

Publication date:

2004-01

Permanent link:

https://doi.org/10.3929/ethz-b-000052221

\section{Rights / license:}

Creative Commons Attribution 4.0 International

\section{Originally published in:}

The European Physical Journal C 32(2), https://doi.org/10.1140/epjc/s2003-01382-2 


\title{
Searching for evolutions from pure states into mixed states with entangled neutral kaons
}

\author{
H.-J. Gerber \\ Institute for Particle Physics, ETH, 8093 Zürich, Switzerland
}

Received: 14 March 2003

Published online: 26 November 2003 - (c) Springer-Verlag / Società Italiana di Fisica 2003

\begin{abstract}
Entangled two-quantum states may be a sensitive probe for a loss of quantum coherence due to apparent violations of quantum mechanics, e.g. as caused by gravitation. We show that there exists a modest experiment sensitive to any one of the nine phenomenological parameters which describe decoherence in the neutral kaon system.
\end{abstract}

Gravitation might influence the coherence of wave functions and thereby create transitions from pure states to mixed states [1]. This could appear as a violation of quantum mechanics (QMV). Suitably entangled neutral kaons can serve to detect decoherence. The kaon is a two-state system which is described by a $2 \times 2$ density matrix. Its time development has, in general, 16 real parameters. Quantum mechanics (QM) requires nine of them to vanish [2].

This work describes the principle of an exploratory measurement, using entangled neutral kaons, that has a selective sensitivity to all of these nine parameters.

It has been shown before that there exists a set of experiments to determine all of them [3] at the level of expectations of some models of quantum gravity $[2,4]$. The measurement discussed here is experimentally relatively modest. It exploits the existence of non-linear inequalities that generally guarantee positive probabilities, first applied to the decoherent kaon system in [5].

The general subject of decoherence in the neutral kaon system has been treated before with restricted decoherence parameter sets $[2,4-10]$. As our approach and also the approximation method differ from the ones in the neutral kaon literature, we give some general introduction.

The quantum-mechanical time evolution of a neutral $K$ meson is given by the four (complex) elements $U^{i j}(t)(i, j=$ 1,2 ), of the (non-singular) matrix $U(t)$, with respect to the basis $(K \bar{K})$, of the form $\Psi(t)=U(t) \Psi(0)$. $\Psi$ has two components, the amplitude for the meson to be a kaon, and the amplitude to be an antikaon, and it represents a pure state. The density matrix $\rho(t)$, which, in general, describes a mixed state, evolves like $\Psi \Psi^{+}$, i.e. as

$$
\rho(t)=U(t) \rho(0) U^{+}(t), \quad t \geq 0 .
$$

The form of this equation grants the conservation of

(i) the rank, and

(ii) the positivity of $\rho(t)$.
Property (i) makes a pure state (which is characterized by rank $(\rho(0))=1$ ) to stay pure, and (ii) avoids the occurrence of negative values for probabilities. For the traces we require

(iii) $\operatorname{tr}(\rho(t>0)) \leq \operatorname{tr}(\rho(0)) \leq 1$ to avoid values for probabilities to exceed one. Due to the decay of the $K$ meson, $U(t)$ is not unitary.

In order to derive a parameterization of $\rho \equiv \rho(t)$ which will enable one to separate the effects of QM and QMV, we represent $2 \times 2$ matrices as linear combinations of the Pauli matrices $\sigma^{\mu}, \mu=0, \ldots, 3$ (with $\sigma^{0}=$ unit matrix) with real coefficients $R^{\mu}: \rho=R^{\mu} \sigma^{\mu}$.

We note that the determinant is $|\rho|=R^{\mu} R_{\mu} \equiv R^{0} R^{0}-$ $R^{1} R^{1}-R^{2} R^{2}-R^{3} R^{3}$. Denoting $\rho_{0} \equiv \rho(0)$ and $\rho_{0}=R_{0}^{\mu} \sigma^{\mu}$, we see that (1) induces $R^{\mu} R_{\mu}=\|U\|^{2} R_{0}^{\mu} R_{0 \mu}$, i.e. a multiple of a Lorentz transformation [3] $R=\Lambda(t) R_{0}$ between the four-vectors $R \equiv\left(R^{\mu}\right)$ and $R_{0} \equiv\left(R_{0}^{\mu}\right)$. (We note in passing that we make use of the homomorphism of the unimodular group $S L(2, C)$ onto the proper Lorentz group.) $\|U\|$ is the absolute value of $\operatorname{det}(U)$. As pure states are characterized by $R^{\mu} R_{\mu}=0$ ( $R$ light-like), the Lorentz transformation lets pure states stay pure.

Invariance with respect to time translation $R(t+h)=$ $\Lambda(h) R(t)$ requires $\Lambda(t)=\mathrm{e}^{T t}$, where $T=\left(T^{\mu \nu}\right)=T^{00} \mathbf{1}_{4 \times 4}+$ $L$, and where $L$ is an element of the Lie algebra of the Lorentz transformations, and thus satisfies

$$
g L g=-L^{\mathrm{T}}
$$

$g$ is a $4 \times 4$ matrix with $g^{00}=-g^{11}=-g^{22}=-g^{33}=$ $1, g^{\alpha \beta}=0$ for $\alpha \neq \beta$; ()$^{\mathrm{T}}$ is the transpose of (). We will explicitly use

$$
L=\left(\begin{array}{cccc}
0 & N^{1} & N^{2} & N^{3} \\
N^{1} & 0 & -I^{3} & I^{2} \\
N^{2} & I^{3} & 0 & -I^{1} \\
N^{3} & -I^{2} & I^{1} & 0
\end{array}\right) \approx\left(\begin{array}{cccc}
0 & -\gamma & 0 & 0 \\
-\gamma & 0 & 0 & 0 \\
0 & 0 & 0 & \omega \\
0 & 0 & -\omega & 0
\end{array}\right)
$$


The last term in (3) anticipates the empirical result that, for neutral kaons, the values of the parameters $N^{1}$ and $I^{1}$ are dominating those of the other ones.

(As a side remark we mention that short calculations (not presented here) reveal the physical significance of the parameters $T^{00}, N^{i}$, and $I^{i}$ as follows: Denoting $\gamma=-N^{1}$ and $\omega=-I^{1}, \omega$ is the angular frequency of the strangeness oscillation between $K$ and $\bar{K}$, and $T^{00} \pm \gamma$ are the decay rates of the $C P$ eigenstates $(K \pm \bar{K}) / 2^{1 / 2}$. $C P$ violation is expressed by $I^{2}$ and $N^{2}$.)

A violation of the quantum-mechanical time development $(\mathrm{QMV})$ is obtained by the use of the most general $4 \times 4$ matrix for $T$ :

$$
T=T^{00} \mathbf{1}_{4 \times 4}+L+X,
$$

with

$$
\begin{aligned}
X & =\left(\begin{array}{cccc}
0 & S^{1} & S^{2} & S^{3} \\
-S^{1} & -J^{1} & D^{3} & D^{2} \\
-S^{2} & D^{3} & -J^{2} & D^{1} \\
-S^{3} & D^{2} & D^{1} & -J^{3}
\end{array}\right) \\
& \equiv\left(\begin{array}{cccc}
0 & x_{4} & x_{5} & x_{6} \\
-x_{4} & -x_{1} & -x_{7} & -x_{8} \\
-x_{5} & -x_{7} & -x_{2} & -x_{9} \\
-x_{6} & -x_{8} & -x_{9} & -x_{3}
\end{array}\right),
\end{aligned}
$$

which satisfies

$$
g X g=+X^{\mathrm{T}} .
$$

When $X \neq(0), T$ of (4) is no more the generator of a transformation which leaves $R^{\mu} R_{\mu}=0$ invariant, and thus pure states become mixed ones. It is the different signs in (2) and (6) that will prove to be crucial for the experimental distinction of effects of QMV from those of QM.

For the probability of a single neutral kaon with the density matrix $\rho_{0}=R_{0}^{\mu} \sigma^{\mu}$ at time $t=0$ to become a neutral kaon with the density matrix $\rho_{f}=R_{f}^{\mu} \sigma^{\mu}$ at time $t$, we obtain

$$
W(t)=\operatorname{tr}\left(\rho_{f} \rho(t)\right)=2 R_{f}^{\mu}\left(\mathrm{e}^{T t}\right)^{\mu \nu} R_{0}^{\nu} \equiv 2 R_{f}^{\mathrm{T}}\left(\mathrm{e}^{T t}\right) R_{0} .
$$

$W(t)$ in $(7)$ is understood as the probability that a measuring device whose sensitivity is characterized by the matrix $\rho_{f}$, would respond to a kaon presented in the state $\rho(t)$. The last term in (7) expresses the one before in $4 \times 4$-matrix notation. $R_{f}^{\mathrm{T}}=\left(R_{f}^{\mu}\right)^{\mathrm{T}}$ and $R_{0}=\left(R_{0}^{\nu}\right)$ are 4-component arrays.

A pair of $K$ mesons in a general (mixed) state is described by a positive semidefinite $4 \times 4$ density matrix $\rho\left(t_{1}, t_{2}\right)$ with respect to the basis $(K K, K \bar{K}, \bar{K} K, \bar{K} \bar{K})$, and it is assumed to evolve like $\rho\left(t_{1}\right) \otimes \rho\left(t_{2}\right)[11]$. (With the two times $t_{1}, t_{2}$ we anticipate that later measurements on the individual particles will generally be performed at different times $t_{1}, t_{2}$.) If we develop the $4 \times 4$ density matrices in terms of the products $\left(\sigma^{\mu} \otimes \sigma^{\nu}\right)$ with coefficients $R^{\mu \nu} \equiv R^{\mu \nu}\left(t_{1}, t_{2}\right), \quad R_{0}^{\mu \nu} \equiv R^{\mu \nu}(0,0)$, as follows:

$$
\rho\left(t_{1}, t_{2}\right)=R^{\mu \nu}\left(\sigma^{\mu} \otimes \sigma^{\nu}\right),
$$

we obtain

$$
R^{\mu \nu}=\left(\mathrm{e}^{T t_{1}}\right)^{\mu \alpha} R_{0}^{\alpha \beta}\left(\mathrm{e}^{T t_{2}}\right)^{\nu \beta} .
$$

The probability to detect the state with the density matrix $\rho_{f}=R_{f}^{\mu \nu}\left(\sigma^{\mu} \otimes \sigma^{\nu}\right)$ is

$$
\begin{aligned}
& W\left(t_{1}, t_{2}\right)=\operatorname{tr}\left(\rho_{f} \rho\left(t_{1}, t_{2}\right)\right)=4 R_{f}^{\mu \nu} R^{\mu \nu} \\
& =4 R_{f}^{\mu \nu}\left(\mathrm{e}^{T t_{1}}\right)^{\mu \alpha} R_{0}^{\alpha \beta}\left(\mathrm{e}^{T t_{2}}\right)^{\nu \beta} \\
& =4 R_{f}^{\mu \nu}\left(\mathrm{e}^{(L+X) t_{1}}\right)^{\mu \alpha} R_{0}^{\alpha \beta}\left(g \mathrm{e}^{(-L+X) t_{2}} g\right)^{\beta \nu} \\
& \quad \times \exp \left(T^{00}\left(t_{1}+t_{2}\right)\right) \\
& \equiv 4 \operatorname{Tr}\left(\boldsymbol{R}_{f}^{\mathrm{T}} \mathrm{e}^{(L+X) t_{1}} \boldsymbol{R}_{0} g \mathrm{e}^{(-L+X) t_{2}} g\right) \\
& \quad \times \exp \left(T^{00}\left(t_{1}+t_{2}\right)\right) .
\end{aligned}
$$

The last equation in (10) expresses the one before by $4 \times 4$ matrices. The elements of $\boldsymbol{R}_{0}$ and $\boldsymbol{R}_{f}$, e.g., are respectively, $R_{0}^{\alpha \beta}$ and $R_{f}^{\nu \mu}$. Tr acts on their superscripts.

The difference of the relative signs of $L$ and $X$ in the two exponentials in (10) is the empirical consequence of the different signs in (2) and (6).

Equations (9) and (10) do not any more grant the positivity of $\rho\left(t_{1}, t_{2}\right)$ and of $W\left(t_{1}, t_{2}\right)$ unless the generalization from QM to QMV, i.e. the introduction of decoherence, is realized in a very specific (and, as will be seen, in an astonishingly simple) way in (1), which in turn leads to restrictions for the values of the parameters $X$.

Such restrictions are not existent within QM.

We may write $(1)$ as $\rho(t)=\Phi(\rho(0)), t \geq 0$, where $\Phi(\bullet)$ is the linear map which describes the single particle evolution. It has been emphasized that the positivity of this map does not grant the positivity of the twoparticle quantities $\rho\left(t_{1}, t_{2}\right)$ and $W\left(t_{1}, t_{2}\right)$, but that the complete positivity of $\Phi(\bullet)$ is sufficient [12]. Based on a specific example we can see that complete positivity is also a necessary condition. Consider the neutral kaon pair state $\Psi^{+}=(K K+\bar{K} \bar{K}) / \sqrt{2}$, which is produced in $p \bar{p}$ annihilation, and which is, at time $t=0$, described by

$$
\rho(0,0)=1 / 2 E_{i k} \otimes E_{i k}=1 / 2\left(\begin{array}{llll}
1 & 0 & 0 & 1 \\
0 & 0 & 0 & 0 \\
0 & 0 & 0 & 0 \\
1 & 0 & 0 & 1
\end{array}\right)
$$

where the matrices $E_{i k}(i, k=1,2)$, have elements $\left(E_{i k}\right)^{m n}=\delta^{i m} \delta^{k n}$. In order to calculate probabilities related to measurements of particle 1 at time $t=0$ and of particle 2 at time $t_{2}$, we need

$$
\rho\left(0, t_{2}\right)=1 / 2 E_{i k} \otimes \Phi\left(E_{i k}\right)=1 / 2\left(\begin{array}{l}
\Phi\left(\begin{array}{ll}
1 & 0 \\
0 & 0
\end{array}\right) \Phi\left(\begin{array}{ll}
0 & 1 \\
0 & 0
\end{array}\right) \\
\Phi\left(\begin{array}{ll}
0 & 0 \\
1 & 0
\end{array}\right) \Phi\left(\begin{array}{ll}
0 & 0 \\
0 & 1
\end{array}\right)
\end{array}\right) .
$$


As this state does occur in the laboratory, $\rho\left(0, t_{2}\right)$ has to be a positive matrix.

According to [13], Theorem 2, the positivity of $E_{i k} \otimes$ $\Phi\left(E_{i k}\right)$ is just the condition, necessary and sufficient, for the linear map $\Phi(\bullet)$ to be completely positive, i.e. to be of the form $\Phi(\bullet)=U_{i} \bullet U_{i}^{+}$, with four $2 \times 2$ matrices $U_{i}$, suitably normalized. Equation (1), generalized to QMV, becomes

$$
\rho(t)=\Phi(\rho(0))=U_{i}(t) \rho(0) U_{i}^{+}(t) .
$$

Developing $U_{i}(t)=U_{i}^{\mu} \sigma^{\mu}\left(U_{i}^{\mu}\right.$ complex $)$, we now find

$$
T^{\mu \nu}=a^{\alpha \beta} \operatorname{tr}\left(\sigma^{\mu} \sigma^{\alpha} \sigma^{\nu} \sigma^{\beta}\right) / 2
$$

with $a^{\alpha \beta}=\mathrm{d} / \mathrm{d} t\left(U_{i}^{\alpha} U_{i}^{\beta *}\right)_{t=0}=a^{\beta \alpha *}$. With the initial condition $U_{i}^{\alpha}(0) U_{i}^{\beta *}(0)=\delta^{\alpha 0} \delta^{\beta 0}, \alpha, \beta=0, \ldots, 3$, the submatrix $\left(a^{m n}\right), m, n=1, \ldots, 3$ is seen to be positive (semidefinite). We note the following relations: $N^{i}=$ $2 \operatorname{Re} a^{0 i}$ and $I^{i}=2 \operatorname{Im} a^{0 i}, J^{i}=2\left(a^{j j}+a^{k k}\right)$ (no sum $j j, k k)$, and $D^{i}=2 \operatorname{Re} a^{j k}, S^{i}=2 \operatorname{Im} a^{j k}, i j k$ is an even permutation of 123 . Besides the requirement $\operatorname{det}\left(\left(a^{m n}\right)\right) \geq$ 0 , which constitutes a cubic inequality for the $J^{i}, D^{i}, S^{i}$, (13) to (15) grant the positivity of $\left(a^{m n}\right)$ :

$$
\begin{gathered}
\left(D^{i}\right)^{2}+\left(S^{i}\right)^{2} \leq\left(\left(J^{i}\right)^{2}-\left(J^{j}-J^{k}\right)^{2}\right) / 4 \\
J^{i} \geq 0 \quad \forall i=1, \ldots, 3 \\
J^{i} \leq J^{j}+J^{k}
\end{gathered}
$$

( $i j k$ is a permutation of 123). A special case of (13) (which reminds one of the classical moments of inertia) has been given before $[5,14]$.

About the matrix $X$ we note the following. If any one of the three diagonal elements, $J^{i}$, vanishes, then the other two ones are equal, and all off-diagonal elements, $D^{i}$ and $S^{i}$, vanish, and if any two of the three diagonal elements, $J^{i}$, vanish, then all elements of $X$ vanish.

For $\rho(t)$ to satisfy also $\operatorname{tr}(\rho(t>0)) \leq \operatorname{tr}(\rho(0)) \leq 1$ demands the additional conditions

$$
\begin{aligned}
T^{00} & \leq 0 \\
\left(T^{00}\right)^{2} & \geq\left(N^{1}+S^{1}\right)^{2}+\left(N^{2}+S^{2}\right)^{2}+\left(N^{3}+S^{3}\right)^{2} .
\end{aligned}
$$

To summarize, (3), (4), (5), (7) and (10) with the conditions (13)-(17), and with $\operatorname{det}\left(\left(a^{m n}\right)\right) \geq 0$, describe a time evolution where $\rho(t)$ has the properties (ii) and (iii) but not necessarily (i). $\rho(t)$ may thus be "quantum-mechanics violating" as it allows for transitions from pure states to mixed states, depending on the nine parameters $J^{i}, D^{i}$, and $S^{i}$ in the matrix $X$. The six parameters $N^{i}$ and $I^{i}$ in the generating Lorentz matrix $L$ do not violate quantum mechanics. They preserve the purity of states. The $I^{i}$ also preserve $\operatorname{tr}(\rho(t))$, the $N^{i}$ do not.

Equation (17) is an analogue to the well-known relation (derived in [15]) which limits the size of symmetry violations in terms of the measured eigenvalues of $\left(T^{\mu \nu}\right)$. (We mention the crude estimates: $T^{00} \approx-\left(\gamma_{S}+\gamma_{L}\right) / 2$ and $N^{1} \approx\left(\gamma_{L}-\gamma_{S}\right) / 2$. Since $\gamma_{S} \gg \gamma_{L}$, empirically, (17) leaves only little room for the values of the other symmetryviolating parameters, $N^{i}, S^{i}$.)

To further separate QMV and QM terms, we apply [16, $17]$

$$
\mathrm{e}^{(L+X) t}=\mathrm{e}^{L t} \mathrm{e}^{\boldsymbol{D}(t,-L, X)}=\mathrm{e}^{\boldsymbol{D}(t, L, X)} \mathrm{e}^{L t},
$$

with

$$
\boldsymbol{D}(t, L, X) \equiv \int_{0}^{t} \mathrm{~d} \tau \mathrm{e}^{L \tau} X \mathrm{e}^{-L \tau}=-\boldsymbol{D}(-t,-L, X),
$$

to $(10)$ :

$$
\begin{aligned}
& W\left(t_{1}, t_{2}\right) \\
& =4 \operatorname{Tr}\left(\mathrm{e}^{L t_{1}} \boldsymbol{R}_{0} g \mathrm{e}^{-L t_{2}} \mathrm{e}^{\boldsymbol{D}\left(t_{2}, L, X\right)} g \boldsymbol{R}_{f}^{\mathrm{T}} \mathrm{e}^{\boldsymbol{D}\left(t_{1}, L, X\right)}\right) \\
& \quad \times \exp \left(T^{00}\left(t_{1}+t_{2}\right)\right) .
\end{aligned}
$$

Equation (20) summarizes the measurements of the 16 parameters of the $K \bar{K}$ pair. It is the general expression for the frequency of occurrence for the two particles to be detected in the final state $\rho\left(t_{1}, t_{2}\right)$, which have originally been prepared in the state $\rho(0,0)$.

For practical evaluations of $W\left(t_{1}, t_{2}\right)$ we use the approximation for $L$ indicated in (3), and we obtain

$\mathrm{e}^{L t}=\left(\begin{array}{cc}\sigma^{0} \cosh (\gamma t)-\sigma^{1} \sinh (\gamma t) & (0) \\ (0) & \sigma^{0} \cos (\omega t)+\mathrm{i} \sigma^{2} \sin (\omega t)\end{array}\right)$.

Since, apart from $T^{00}, \gamma$, and $\omega$, all the other parameters are small, we thus include them in $X$. The values of the elements of $\mathrm{e}^{\boldsymbol{D}(t, L, X)}$, for $t \gtrsim\left|1 / T^{00}\right|$, will then always be small, and we may set

$$
\mathrm{e}^{\boldsymbol{D}} \approx \mathbf{1}+\boldsymbol{D}+\ldots+(1 / n !) \boldsymbol{D}^{n} .
$$

As $\boldsymbol{D}$ is a linear function of the small parameters $x_{k}, \boldsymbol{D}=$ $x_{k} \boldsymbol{D}_{k}$, with $\boldsymbol{D}_{k} \equiv\left(\partial / \partial x_{k}\right) \boldsymbol{D}(t, L, X)=\boldsymbol{D}\left(t, L, X_{k}\right),(21)$ includes all terms including the $n$th order in $x_{k}$. We have set $X=x_{k} X_{k}$, where $X_{k}$ may be read off from (5).

Explicit general expressions for $W\left(t_{1}, t_{2}\right)$ are long, but may now be readily calculated to any order. A selection has been given before $[8,18]$ for restricted parameter sets.

For the measurement to be discussed the following considerations are of relevance.

Pairs of kaons in the exact (pure, entangled) "strangeness-singlet" state $\Psi^{-}=(K \bar{K}-\bar{K} K) / \sqrt{2}$ are copiously available in the laboratory. Their density matrix is [11] $\rho(0,0)=\left(\sigma^{\mu} \otimes \sigma_{\mu}\right) / 4$, and thus $R_{0}^{\alpha \beta}=g^{\alpha \beta} / 4$. As this is a Lorentz-invariant tensor, its quantum-mechanical time development $(X=0)$ for equal times $t_{1}=t_{2}=t$, yields $R^{\mu \nu}=c g^{\mu \nu}$, where $c$ is a numerical factor. Apart from the decay this state is thus invariant with time. (An analogon to this property is the fact that a pair of particles of spin $1 / 2$, in the singlet state, does not precess in an external magnetic field [22].)

The two particles shall be observed by spatially separated and independent devices whose sensitivities are described by matrices $\rho_{1}$ and $\rho_{2}$. The measurement is thus 
represented by $\rho_{f}=\rho_{1} \otimes \rho_{2}=R_{1}^{\mu} R_{2}^{\nu}\left(\sigma^{\mu} \otimes \sigma^{\nu}\right)$, with $R_{f}^{\mu \nu}=R_{1}^{\mu} R_{2}^{\nu}$, or in matrix notation

$$
\boldsymbol{R}_{f}=R_{1} R_{2}^{\mathrm{T}} \quad \text { (independent measurements). }
$$

Insertion into (20) yields

$$
\begin{aligned}
& W_{1}\left(t_{1}, t_{2}\right) \\
& =4 R_{1}^{\mathrm{T}} \mathrm{e}^{\boldsymbol{D}\left(t_{1}, L, X\right)} \mathrm{e}^{L t_{1}} \boldsymbol{R}_{0} g \mathrm{e}^{-L t_{2}} \mathrm{e}^{\boldsymbol{D}\left(t_{2}, L, X\right)} g R_{2} \\
& \quad \times \exp \left(T^{00}\left(t_{1}+t_{2}\right)\right) .
\end{aligned}
$$

With the initial state $\Psi^{-}$, and with $\boldsymbol{R}_{0}=g / 4,(23)$ becomes

$$
\begin{aligned}
& W_{2}\left(t_{1}, t_{2}\right) \\
& =R_{1}^{\mathrm{T}} \mathrm{e}^{\boldsymbol{D}\left(t_{1}, L, X\right)} \mathrm{e}^{L\left(t_{1}-t_{2}\right)} \mathrm{e}^{\boldsymbol{D}\left(t_{2}, L, X\right)} g R_{2} \\
& \quad \times \exp \left(T^{00}\left(t_{1}+t_{2}\right)\right) .
\end{aligned}
$$

The probability to observe identical pure final states $\left(\rho_{1}=\right.$ $\left.\rho_{2}=\rho, R_{1}=R_{2}=R=\left(R^{\mu}\right)\right)$ at the same time $\left(t_{1}=t_{2}=\right.$ $t$ ), of particles having originated from $\Psi^{-}$is

$$
W_{3}(t, t)=R^{\mathrm{T}} \mathrm{e}^{2 \boldsymbol{D}(t, L, X)} g R \exp \left(2 T^{00} t\right),
$$

or, up to first order,

$$
W_{4} \approx 2 x_{k} R^{\mathrm{T}} \boldsymbol{D}\left(t, L, X_{k}\right) g R \quad \exp \left(2 T^{00} t\right) .
$$

Equation (26) together with the constraints (13)-(15), offer the possibility for a simplified exploratory experiment to find out whether any one of the $x_{k}$ differs from zero.

The experiment consists of two measurements of the type $W_{4}$ : the probabilities for the state $\Psi^{-}$to develop into the pair $K K$ or to develop into the pair $\bar{K} \bar{K}$, designated as $W(K K)$ or $W(\bar{K} \bar{K})$, respectively. Inserting $R_{K K}=$

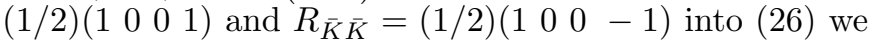
obtain linear combinations of the $\mu \nu$-elements of $\boldsymbol{D}_{k}, \boldsymbol{D}_{k}^{\mu \nu}$. Explicitly, we find (up to first order in $x_{k}$ )

$$
\begin{aligned}
W(K K \text { or } \bar{K} \bar{K})= & x_{k} \exp \left(2 T^{00} t\right) \\
& \times\left(\boldsymbol{D}_{k}^{00} \mp \boldsymbol{D}_{k}^{03} \pm \boldsymbol{D}_{k}^{30}-\boldsymbol{D}_{k}^{33}\right)
\end{aligned}
$$

and note the simplicity of the combination

$$
\begin{aligned}
W^{+} & \equiv W(K K)+W(\bar{K} \bar{K}) \\
& =2 x_{k} \exp \left(2 T^{00} t\right)\left(\boldsymbol{D}_{k}^{00}-\boldsymbol{D}_{k}^{33}\right) \equiv x_{k} f_{k}(t) .
\end{aligned}
$$

We now show, by explicit calculation, that any combination of non-vanishing parameters $x_{1}$ to $x_{9}$, which satisfy (13)(15), creates a signal in the observable $W^{+}$.

In

$$
\begin{gathered}
W^{+}=x_{k} f_{k}(t)=x_{1} f_{1}(t)+x_{2} f_{2}(t)+x_{3} f_{3}(t) \\
+x_{4} f_{4}(t)+x_{9} f_{9}(t),
\end{gathered}
$$

the functions

$$
f_{1}(t)=1 / 4 \exp \left(2 T^{00} t\right)(-2 \gamma t+\sinh (2 \gamma t)) / \gamma,
$$

$$
\begin{aligned}
& f_{2}(t)=1 / 4 \exp \left(2 T^{00} t\right)(2 \omega t-\sin (2 \omega t)) / \omega, \\
& f_{3}(t)=1 / 4 \exp \left(2 T^{00} t\right)(2 \omega t+\sin (2 \omega t)) / \omega, \\
& f_{4}(t)=\exp \left(2 T^{00} t\right)(\sinh (\gamma t))^{2} / \gamma
\end{aligned}
$$

and

$$
f_{9}(t)=-\exp \left(2 T^{00} t\right)(\sin (\omega t))^{2} / \omega
$$

are seen to be independent from each other. A measurement over a suitable interval of $t$ can thus only show a vanishing $W^{+}$, if $x_{1}$ to $x_{4}$ and $x_{9}$ vanish. Then, due to (13)-(15), all the values of $x_{1}$ to $x_{9}$ have to vanish.

The experiment consists in the production of entangled $K \bar{K}$ pairs in the strangeness-singlet state $\Psi^{-}$, readily available in $\phi$ decays [19] or in antiproton annihilations in hydrogen under suitable conditions [20], and in the subsequent observation of their semileptonic decays, which, due to the validity of the $\Delta S=\Delta Q$ rule [21], indicate the kaons' strangenesses just before they decayed. Useful events are those with similar decay times. By a proper choice of the events, $W^{+}$can then be evaluated.

In summary we have described the principle of a measurement of a quantity, $W^{+}$, sensitive to decoherence processes in the time evolution of the two-particle system of separated, entangled $K$ and $\bar{K}$, in vacuum. Such processes are QM-forbidden, $C P T$ violating, but allowed in certain models of quantum gravity. From our phenomenological viewpoint there exist nine experimentally distinguishable manifestations of transitions from pure states to mixed states. If $W^{+}$is found not to vanish, then two of these, at least, have to arise in reality.

Acknowledgements. The author is indebted to M. Fidecaro and R. Floreanini for enlightening comments.

\section{References}

1. S.W. Hawking, Commun. Math. Phys. 87, 395 (1982)

2. J. Ellis, J.S. Hagelin, D.V. Nanopoulos, M. Srednicki, Nucl. Phys. B 241, 381 (1984)

3. H.-J. Gerber, Phys. Rev. Lett. 80, 2969 (1998)

4. J. Ellis, J.L. Lopez, N.E. Mavromatos, D.V. Nanopoulos, Phys. Rev. D 53, 3846 (1996)

5. F. Benatti, R. Floreanini, Nucl. Phys. B 488, 335 (1997); F. Benatti, R. Floreanini, R. Romano, J. Phys. A 35, 4955 (2002)

6. J. Ellis, N.E. Mavromatos, D.V. Nanopoulos, Phys. Lett. B 293, 142 (1992)

7. CPLEAR Collaboration, J. Ellis et al., Phys. Lett. B 364, 239 (1995)

8. P. Huet, M. Peshkin, Nucl. Phys. B 434, 3 (1995)

9. CPLEAR Collaboration, R. Adler et al., Phys. Lett. B 286, 180 (1992)

10. F. Benatti, R. Floreanini, Phys. Lett. B 401, 337 (1997)

11. C.P. Enz, R.R. Lewis, Helv. Phys. Acta 38, 860 (1965), reprinted in $C P$ Violation, edited by L. Wolfenstein (North Holland, Amsterdam 1989)

12. F. Benatti, R. Floreanini, Modern Phys. Lett. A 12, 1465 (1997) 
13. Man-Duen Choi, Linear Alg. Appl. 10, 285 (1975)

14. V. Gorini, A. Kossakowski, E.C.G. Sudarshan, J. Math. Phys. 17, 821 (1976)

15. T.D. Lee, R. Oehme, C.N. Yang, Phys. Rev. 106, 340 (1957)

16. R. Feynman, Phys. Rev. 84, 108 (1951)

17. U. Fano, Revs. Modern Phys. 29, 74 (1957)
18. F. Benatti, R. Floreanini, Nucl. Phys. B 511, 550 (1998)

19. DA $\Phi$ NE Physics Handbook, edited by L. Maiani, G. Pancheri, N. Paver (INFN, Frascati, 1992)

20. CPLEAR Collaboration, R. Adler et al., Phys. Lett. B 403, 383 (1997)

21. CPLEAR Collaboration, A. Angelopoulos et al., Physics Reports 374, 165 (2003)

22. P.K. Kabir in [11], p. 874 